
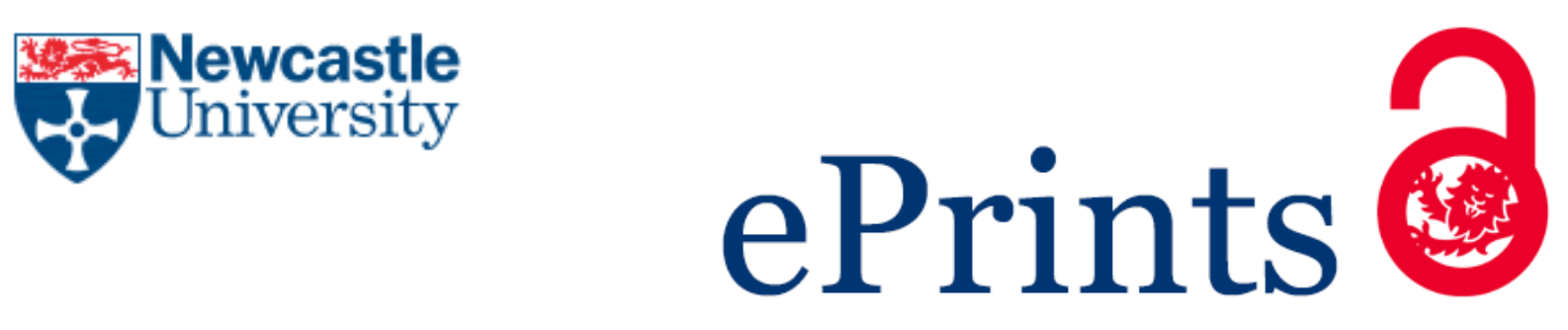

McDonough JR, Law R, Kraemer J, Harvey AP.

Effect of geometrical parameters on flow-switching frequencies in 3D printed

fluidic oscillators containing different liquids.

Chemical Engineering Research and Design 2017, 117, 228-239

Copyright:

(C) 2017. This manuscript version is made available under the CC-BY-NC-ND 4.0 license

DOI link to article:

http://dx.doi.org/10.1016/i.cherd.2016.10.034

Date deposited:

$01 / 03 / 2017$

Embargo release date:

27 October 2017

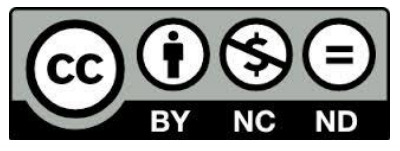

This work is licensed under a

Creative Commons Attribution-NonCommercial-NoDerivatives 4.0 International licence 


\title{
Effect of geometrical parameters on flow-switching frequencies in 3D printed fluidic oscillators containing different liquids
}

\author{
J.R. McDonough ${ }^{\mathrm{a} \dagger}$, R. Law ${ }^{\mathrm{a}}$, J. Kraemer ${ }^{\mathrm{a}}$, A.P. Harvey ${ }^{\mathrm{a}}$

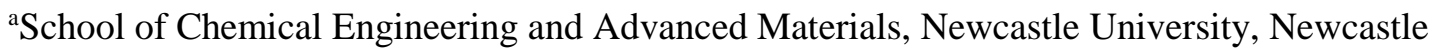 \\ upon Tyne, NE1 7RU \\ ${ }^{\dagger}$ Corresponding Author
}

\begin{abstract}
There is limited information available regarding fluidic oscillator design for liquid phase applications. In this paper, the results of a simple parametric study investigating the effects of seven geometrical parameters on the flow-switching frequencies produced in 3D printed single feedback loop bistable oscillators are reported for a variety of glycerol-water mixtures. The most consequential parameter was the splitter distance (distance between the power nozzle and two outlet streams). Reducing the splitter distance from $10 \mathrm{~mm}$ to $5 \mathrm{~mm}$ produced higher frequencies at the same flow rate. The angle between the outlet channels was also important, with wider angles $\left(18-24^{\circ}\right)$ producing slightly higher frequencies. Feedback loop widths of $4 \mathrm{~mm}$ and greater did not produce flow switching. Other factors that inhibited oscillations were reducing the inlet zone length from $32 \mathrm{~mm}$ to $22 \mathrm{~mm}$ and changing the feedback channel orientation from horizontal to vertical. Increasing the convergence length of the power nozzle (from 5 to $25 \mathrm{~mm}$ ) and changing the feedback loop length (from 101 to 113 $\mathrm{mm}$ ) did not greatly affect the frequencies obtained. Overall, frequencies of $2-22 \mathrm{~Hz}$ were produced for kinematic viscosities of $1.002-4.373 \mathrm{~mm}^{2} / \mathrm{s}$, in the range of $R e=600-12,000$.
\end{abstract}

Keywords: Flow switching, oscillation, fluidic oscillator, 3D printing, OBR, plug flow

\section{Introduction}

Fluidics (fluid logic) is the application of fluid mechanic phenomena to perform logical operations. Fluid oscillators of the bistable amplifier type are one example of fluidics that has found new interest in a wide range of applications. Example gas-phase applications include enhanced microbubble generation in gas spargers [1], flow control, flow separation (aeronautics) [2], noise control [3] and combustion [4]. Basic liquid-phase oscillator applications include sprinklers, shower heads, Jacuzzis and windscreen washers [5]. Recently, improvements in liquid extraction mass transfer coefficients were also reported when using a microchannel oscillator [6,7]. Fluidic oscillators enable autonomous rapid flow switching between two outlets using internal feedback, leading to dual stream pulsations. Switching frequencies ranging from $10 \mathrm{~Hz}$ to $20 \mathrm{kHz}$ [8] are reported, although the highest frequencies are typically only obtainable in microchannels with high Reynolds numbers [9]. The two principle modes of operation are momentum transfer in double feedback designs, and pressure transfer in single feedback loop designs.

The single feedback loop design comprises of a supply port, two outlet channels and two control ports that are connected via a feedback loop (Figure 1). Operation is mainly governed by the Coandă effect, which describes the tendency of a fluid jet emerging from a nozzle to adhere to an adjacent surface. Wall attachment occurs because of the formation of a vortex near the wall as a result of fluid entrainment. This vortex creates a low pressure zone leading to a pressure difference across the jet. Flow switching develops from instabilities provided by the feedback loop, whereby a pressure wave is transferred from the high pressure side to low pressure side (wall attachment side). The resulting feedback flow causes the vortex to grow large enough for the jet to detach and adhere to the other wall. The process is represented in Figure 1. The concave wall between the two outlets shown in Figure 1 also leads to the formation of a secondary stabilisation vortex in the oscillating chamber [10]. 


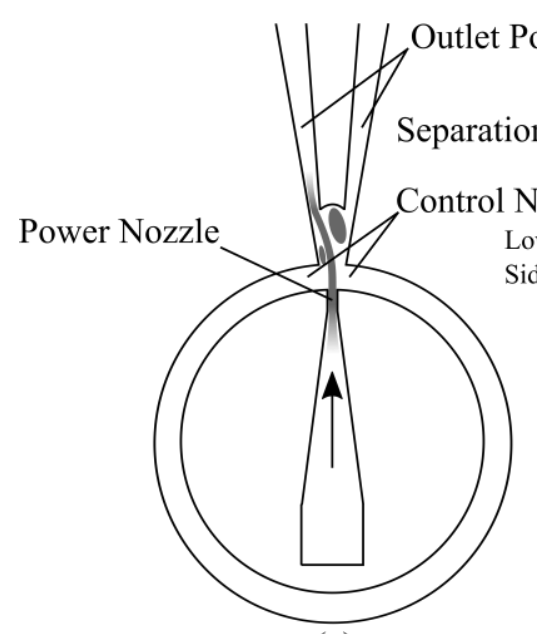

(a)

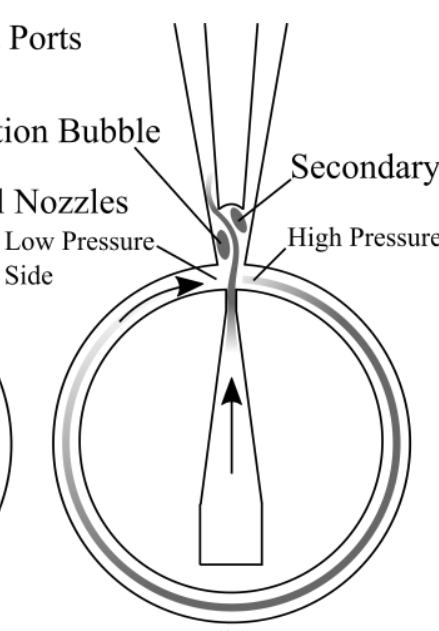

(b)

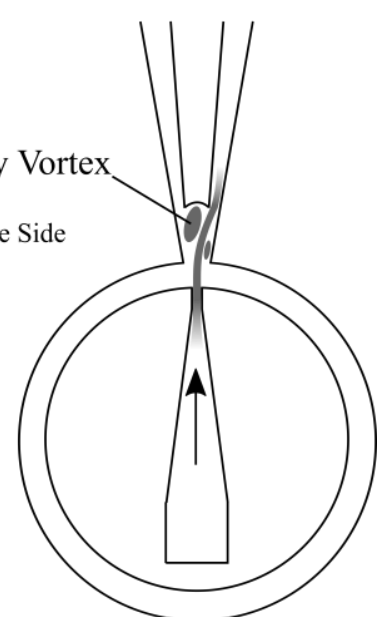

(c)

Figure 1 - Flow switching mechanism in a single feedback loop bistable oscillator; (a) wall attachment and formation of separation bubble, (b) growth of the separation bubble via flow around the feedback channel, (c) switching of the main jet to the other outlet

Fluidic oscillators typically operate under constant Strouhal number [2], defined by equation 1. This is because the frequency response is proportional to the increase in velocity. Tesař et al [11] additionally proposed a modified Strouhal number that enables assessment of the velocity through the feedback loop. As shown in equation 2 , the frequency is the reciprocal of the time taken to complete one full oscillation cycle, in which two propagations around the feedback loop occur. For equation 2 to provide a reliable estimate of the feedback channel velocity, the switching process must occur faster than the separation bubble growth process. In these equations, $f$ is the switching frequency $(H z), b$ is the nozzle width $(m), v$ is the velocity of the jet emerging from the nozzle $(\mathrm{m} / \mathrm{s}), v_{a}$ is the velocity in the feedback channel $(\mathrm{m} / \mathrm{s})$ and $l$ is the length of the feedback channel $(\mathrm{m})$.

$$
\begin{gathered}
S t=\frac{f b}{v} \\
S t^{\prime}=2\left(\frac{l}{b}\right) \frac{f b}{v}=\frac{2 f l}{v}=\frac{v_{a}}{v}
\end{gathered}
$$

The Oscillatory Baffled Reactor operates through a vortex production and dissipation cycle by oscillating the fluid in the presence of baffles. When this mechanism is superimposed onto a net flow rate, plug flow can be realised due to the analogous behaviour of well mixed tanks-in-series. Oscillation has been achieved via two methods in the literature. Typically, the fluid is oscillated using some form of piston and bellows arrangement [12], or through the use of a syringe pump [13]. Alternatively, the baffle assembly itself has been oscillated to induce mixing [14]. Both methods require moving parts, which although is nonproblematic in laboratory settings, may limit their appeal for industrial applications. A potential method for realising mixing and plug flow without the need for moving parts is the application of fluidic oscillators. Fluidic oscillators also offer the potential for enhanced heat and mass transfer rates.

The advantages of fluidic oscillators over conventional oscillator designs (pistons etc) as oscillators for OBRs are their simplicity and passive operation. Passive mixers are preferable because of their robustness. However, research into these oscillators typically focuses on gas phase applications, with only a small number of parametric studies available using for air-based designs $[15,16,8,11]$. The aim of this paper is to investigate the effects of varying fluidic oscillator geometries on the frequency response using liquids of varying densities and viscosities. Here the focus was on the single feedback loop design, as shown in Figure 1.

To accelerate the design, fabrication and characterisation of the fluidic oscillator geometries, 3D printing was used. 3D printing is being used increasingly frequently for such 
rapid prototyping, particularly when it allows development of geometries unobtainable using conventional means. Other advantages include: small batch production, economically viable prototypes, reduced waste, easy customisation and reduced costs.

\section{Methodology}

2.1 Fluidic Oscillator Designs
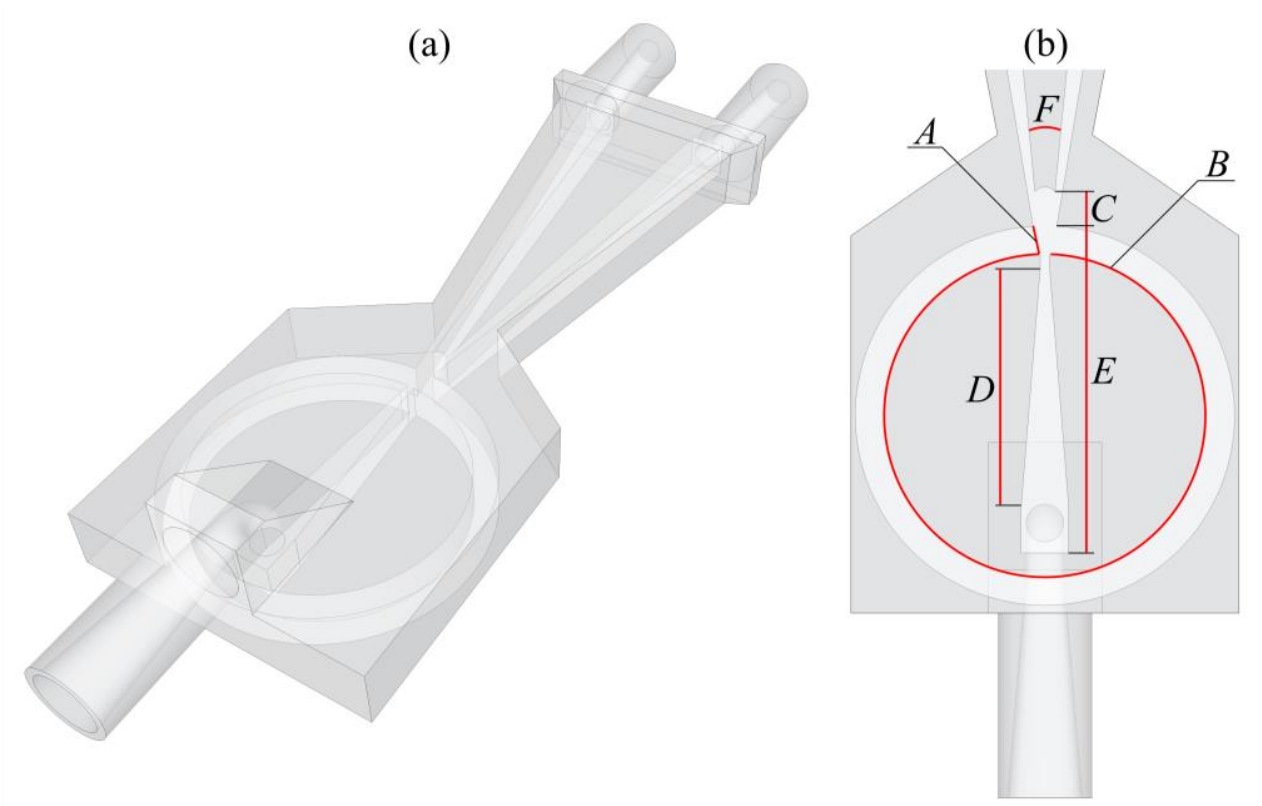

(c)

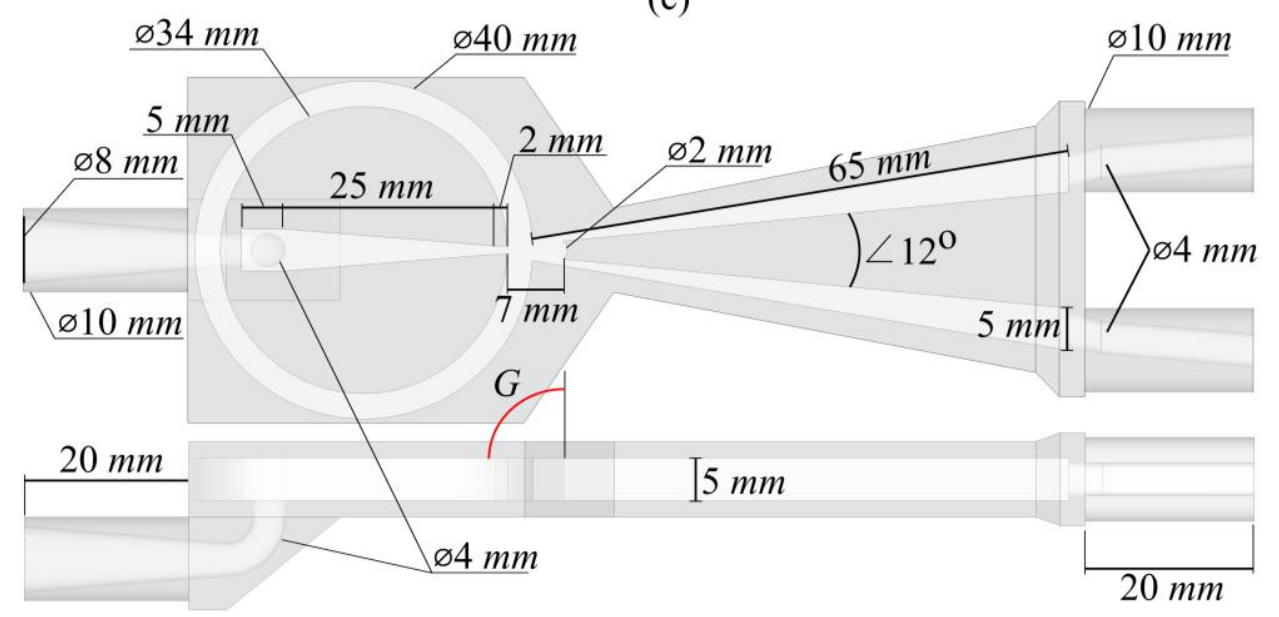

Figure 2 - (a) Fluidic oscillator base design (3D view), (b) geometric parameters, (c) fluidic oscillator base design dimensions

The base design of fluidic oscillator used here is shown above in Figure 2a, which is similar to the model used by Tesař et al [11]. This design consists of a $1 \mathrm{~mm}$ nozzle constriction size and $25 \mathrm{~mm}$ nozzle convergence length, with a total inlet distance of $32 \mathrm{~mm}$. After the nozzle, a splitter with $1 \mathrm{~mm}$ diameter concave wall was positioned at a distance of 7 $\mathrm{mm}$. Also located adjacent to the nozzle were two control ports, connected by a $3 \mathrm{~mm}$ width, $101 \mathrm{~mm}$ length feedback loop. Due to the position of the feedback loop, the liquid was supplied via a $90^{\circ}$ bend, converging from an $8 \mathrm{~mm}$ to $4 \mathrm{~mm}$ tube diameter. The two outlet channels were $65 \mathrm{~mm}$ in length and the diameters of the outlet ports were $4 \mathrm{~mm}$. The external geometry was chosen to minimise the amount of resin required to print each design, while the dimensions of the fluidic oscillator were chosen to be at the same scale as mesoscale oscillatory baffled reactor (which typically consists of a $5 \mathrm{~mm}$ tube). With respect to Figure 
2a, the following parameters were varied in this study (shown in Figure 2b). Each parameter was changed individually, keeping all other factors constant.

- Feedback loop width (A)

- Feedback loop length (B)

- Splitter distance (C)

- Nozzle convergence length (D)

- Inlet zone length (E)

- Outlet channel angle (F)

- Feedback loop orientation $(\mathrm{G})$

\subsection{D Printing}

Two different desktop size stereolithography (SLA) printers were used in the present study, the MiiCraft+ (by MiiCraft) and the Form1+ (by FormLabs). The MiiCraft+ was used to build the majority of the designs in this study. The build specifications are summarised in the following table. SLA technology involves creating each layer using photopolymerisation of a resin by the application of UV light. The MiiCraft+ uses a projected image of the current layer meaning the build time per layer is constant regardless of the object size. Alternatively, the Form1+ uses a single point laser $(155 \mu \mathrm{m}$ size, $120 \mathrm{~mW})$ that tracks quickly over the current layer. Here the print time is dependent on the size of the current layer, but it was found that the Form1+ generally printed quicker than the MiiCraft+, even with multiple models printed simultaneously.

Table 1-3D Printer build specifications

\begin{tabular}{lll}
\hline Printing Function & MiiCraft+ & Form1+ \\
\hline Build Size & $43 \times 27$ x $180 \mathrm{~mm}$ & $125 \times 125 \times 165 \mathrm{~mm}$ \\
XY Resolution & $56 \mu \mathrm{m}$ & $155 \mu \mathrm{m}$ \\
Z-Axis Resolution & $30-100 \mu \mathrm{m}$ & $25-200 \mu \mathrm{m}$ \\
\hline
\end{tabular}

Both printers used similar transparent resins with the following composition: methyl acrylate (55-75 wt\%), methyl acrylate oligomer (35-40 wt\%) and photo-initiator/additives $(10-15 w t \%)$. For the MiiCraft+, it was found that a layer thickness of $75 \mu m$ with curing time of $13 s$ per layer was optimal. This was because higher curing times caused residual resin within the channels to cure, leading to irreversible channel blockages. This problem was not encountered with the Form1+.

The procedure adopted for both printers was as follows: (1) 3D CAD model generation (using Google Sketchup), (2) conversion of the CAD model to a triangular mesh (.stl file format), (3) slicing of the triangular mesh into different layers and conversion of the slices into tool paths for the printer, (4) 3D printing and (5) post-processing. The software used with the MiiCraft+ and Form1+ printers were MiiCraft Builder and PreForm respectively. Post-processing consisted of two steps. The first involved the removal of the support structures from the printed oscillator and the removal of excess resin from the channels using either methanol or propyl alcohol. Then, in the second step, the parts were cured. The MiiCraft+ had a post-curing chamber containing an $18 \mathrm{~W}$ UV bulb (315-400 $\mathrm{nm}$ ) that allowed the parts to be cured within $1 h$. The Form1+ required exposure to sunlight for approximately 24 hours prior to experimentation.

\subsection{Frequency Response Analysis}

The frequency response of the oscillators was measured using piezoelectric pressure transducers (Gems 3500 Series, 0-4 bar.a), with the electric output directly observed using an oscilloscope (Tektronix, TDS 2002C). The pressure transducers were connected at both outlets of the oscillators using Swagelok tee-junctions, which had $10 \mathrm{~mm}$ diameter tube fittings on the through-section and a 1/4 in BSPT fitting for the transducers on the branch. A pressure transducer was also placed at the inlet (using the same tee-junction arrangement) and 
could be monitored using PicoLog via an ADC-20 data logger (but these results are not reported here). Each Swagelok fitting was connected to the fluidic oscillators using PTFE ferrules to avoid damaging the cured resin. Figure 3 shows the placement of the transducers and Swagelok fittings around a fluidic oscillator.

The liquid was supplied to the oscillator from a storage vessel using a gear pump (Greylor PQ-12) powered by a DC power supply (Digimess PM3006) via flexible 1/8 in o.d. tubing. The same tubing was also connected at the two outlet streams, which were recirculated into the storage vessel. The gear pump was calibrated for each fluidic oscillator design by measuring the time taken to fill a $500 \mathrm{~mL}$ container for different pump powers. Each calibration was run in triplicate. A schematic of the experiment set up is shown in Figure 4.

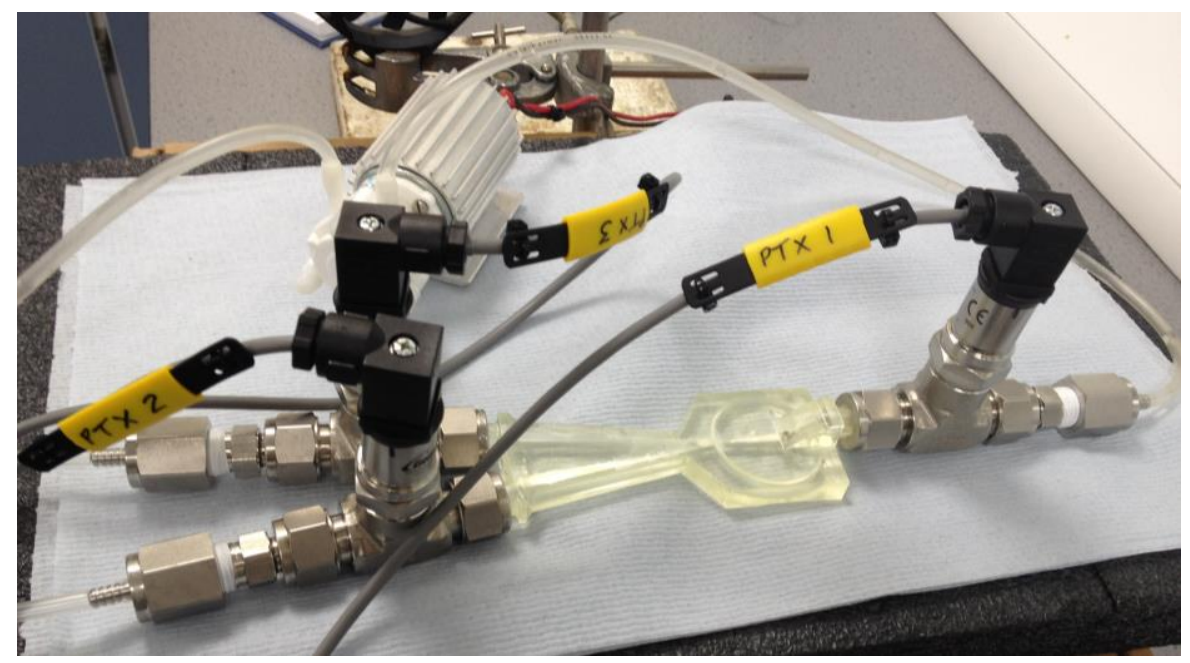

Figure 3 - Pressure transducer configuration around an oscillator

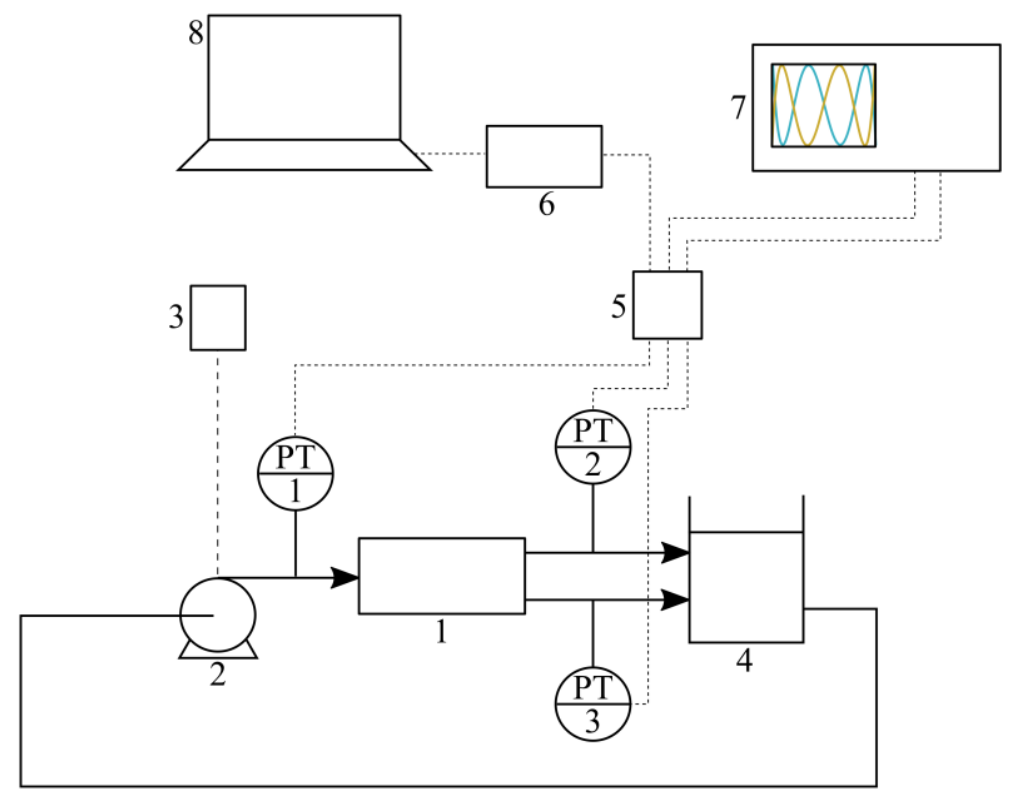

Figure 4-Experiment set-up; (1) fluidic oscillator, (2) gear pump, (3) DC power supply for pump, (4) liquid storage vessel, (5) shared pressure transducer power supply, (6) ADC-20 data logger, (7) oscilloscope, (8) laptop

In addition to studying the effects of different geometric parameters on the switching response, the effects of using different liquids with various densities/viscosities was also investigated. Here, de-ionised water and several mixtures of de-ionised water and glycerol 
(99\%, Sigma Aldrich) were tested (summarised in Table 2). Each was prepared prior to the experiments in $2 L$ batches.

To analyse the frequency response, a simple script was written to perform a fast Fourier transform (FFT) on each recorded waveform from the pressure transducers. The sampling rate of the voltage signal was $500 \mathrm{~Hz}$, giving a Nyquist frequency of $250 \mathrm{~Hz}$. The resulting frequency spectra enabled fast and automated assessment of the switching frequency observed in the experiment. For example, Figure 5 summarises the four types of waveforms observed and their corresponding decomposed frequency spectra. For all designs, the results of waveforms of the type in Figure 5c/d are reported. For waveforms showing multiple frequencies due to the squarer shape of the oscillations, only the first harmonic is reported.

Table 2 - Summary of fluid solutions [17]

\begin{tabular}{cccccccc}
\hline Fluid & $\begin{array}{c}\text { Glycerol } \\
\text { Volume }(L)\end{array}$ & $\begin{array}{c}\text { Water } \\
\text { Volume }(L)\end{array}$ & \%Glycerol & $\begin{array}{c}\text { Density } \\
\left(\mathrm{kg} / \mathrm{m}^{3}\right)\end{array}$ & $\begin{array}{c}\text { Viscosity } \\
(\mathrm{cP})\end{array}$ & $\begin{array}{c}\text { Kinematic } \\
\text { Viscosity } \\
\left(\mathrm{mm}^{2} / \mathrm{s}\right)\end{array}$ & $\begin{array}{c}\text { Relative } \\
R e\end{array}$ \\
\hline 1 & 0 & 2 & 0 & 998 & 1 & 1.00 & 1 \\
2 & 0.2 & 1.8 & 10 & 1024.6 & 1.38 & 1.35 & 0.746 \\
3 & 0.4 & 1.6 & 20 & 1051.2 & 1.99 & 1.89 & 0.532 \\
4 & 0.8 & 1.2 & 40 & 1104.4 & 4.83 & 4.37 & 0.234 \\
5 & 1.4 & 0.6 & 70 & 1184.1 & 35.3 & 29.8 & 0.044 \\
6 & 1.6 & 0.4 & 80 & 1210.7 & 91.8 & 75.9 & 0.013 \\
\hline
\end{tabular}
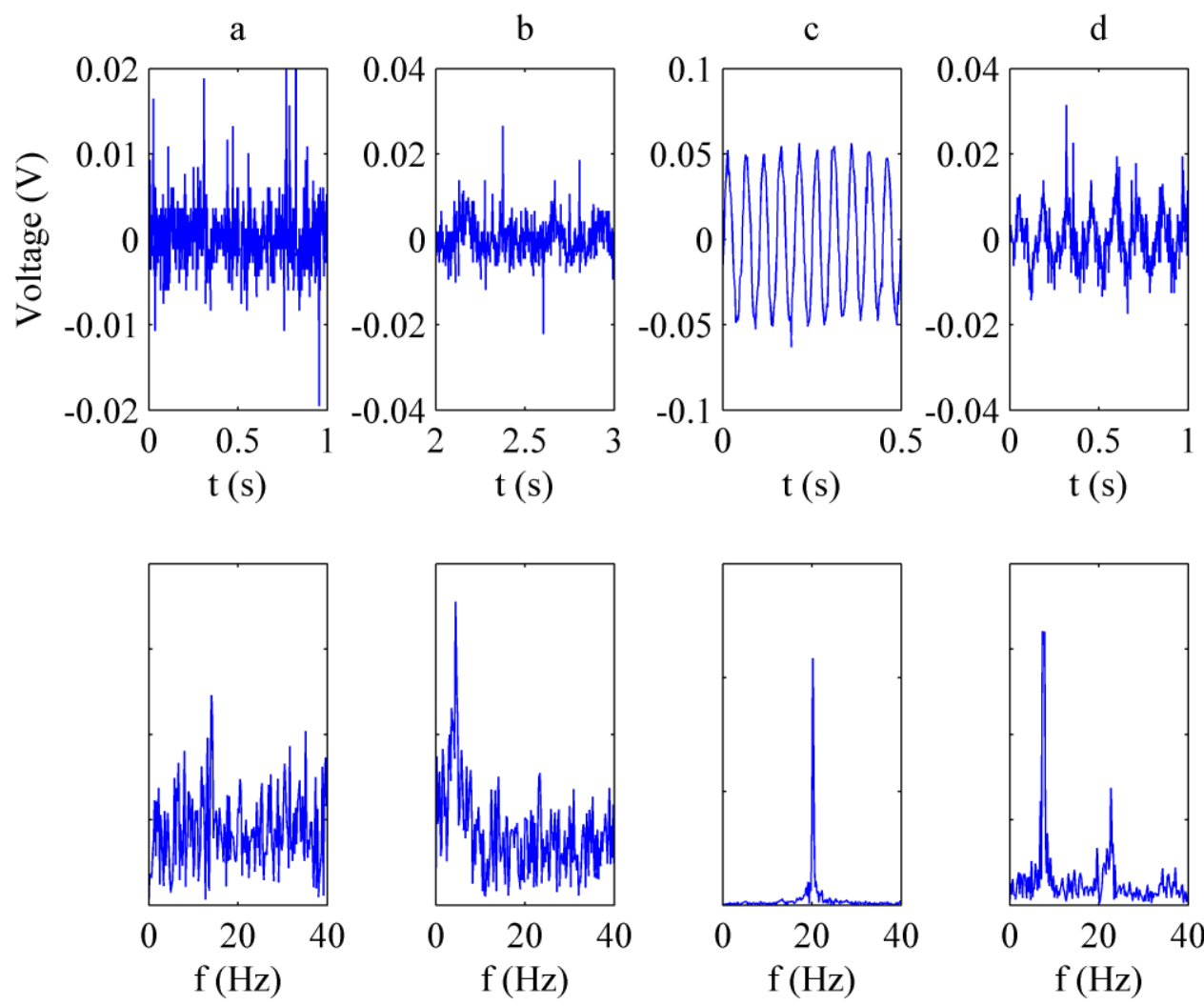

Figure 5 - Sample waveforms from the FFT analysis (top row = recorded waveform, bottom row = corresponding frequency spectrum); (a) no oscillations, (b) sporadic oscillations (just prior to the onset of stable oscillations), (c) stable sinusoidal oscillation, and (d) stable sinusoidal oscillation with second and third harmonic (indicating a squarer waveform) 


\section{Results and Discussions}

\subsection{Flow Rate and Viscosity}

Figure 6 shows the frequencies obtained in the base oscillator design while varying the inlet flow rate and liquid viscosity. At higher flow rates, the frequency gain was reduced resulting in a levelling off of the frequencies observed (causing the response to be linear using a logarithmic $R e$ scale). The exact frequency limit of these oscillators could not be determined with the current experiment methodology.

Liquids with viscosities of $29.8 \mathrm{~mm}^{2} / \mathrm{s}$ and $75.9 \mathrm{~mm}^{2} / \mathrm{s}$ did not oscillate at any of the net flow rates tested. This is presumably because a vortex could not form between the power jet and adjacent wall because of the much lower $R e$. It was found that the frequency was generally independent of the viscosity in the tested range of $1.00-4.37 \mathrm{~mm}^{2} / \mathrm{s}$, and instead was predominately affected by the flow rate.

It was found that $S t$ exhibited a maximum upon increasing $R e$, producing two operating regimes (Figure 6b). This has similarly been observed in air-operated single feedback loop oscillators [11]. Here, St remained constant with shorter tube lengths providing higher frequencies until $R e>3500$, after which $S t$ decreased linearly and became independent of the tube length. This was explained by the propagation velocity in the feedback channel becoming constant, with the frequency limited due to a resonance effect [11]. The exact mechanism of this resonance was not discussed, but is analogous to the phenomenon of vortex shedding frequencies becoming independent of the air flow rate around free cables close to the natural frequency [11].

Figure 6c shows the modified Strouhal number, as proposed by Tesar̆ et al [11]. Although the velocity in the feedback loop continually increases with increasing flow rate, the ratio of the feedback channel to power jet velocities approaches unity before decreasing. In air-based, single-feedback fluidic devices, the upper switching frequency limit is typically associated with a sonic feedback loop velocity: the flow in the feedback channel becomes choked. However, this choked flow condition is unlikely to be the cause of the frequency limit for liquid-filled oscillators because this limit is usually preceded by flashing (vaporisation) and cavitation of the liquid. Instead, the reduction in $S t^{\prime}\left(v_{a} / v\right)$ may be the result of increased pressure loss in the feedback loop at higher velocities, limiting the flow rate gain and thus limiting the rate of growth of the separation bubble.

One mechanism that could explain this is the appearance of secondary flow structures such as Dean vortices in the feedback loop. The Dean number, De, (equation 3) provides a suitable framework to predict the onset of such vortex formation. Assuming the velocity in the feedback channel is $50 \%$ of the jet velocity emerging from the power nozzle (based on Figure 7c), $D e=1,761$ is obtained for a volumetric flow rate of water of $300 \mathrm{~cm}^{3} / \mathrm{min}(R e=$ $2,000)$. This greatly exceeds the theoretical thresholds for Dean vortex formation, around $D e$ $=64-75$ for vortex pairs at $90-145^{\circ}$ from the start of channel curvature [18]. Therefore, as the power jet velocity increases, the accompanying increase in strength of these secondary vortices may limit the feedback channel velocities that can be produced.

$$
D e=\sqrt{\frac{d}{r}} R e
$$




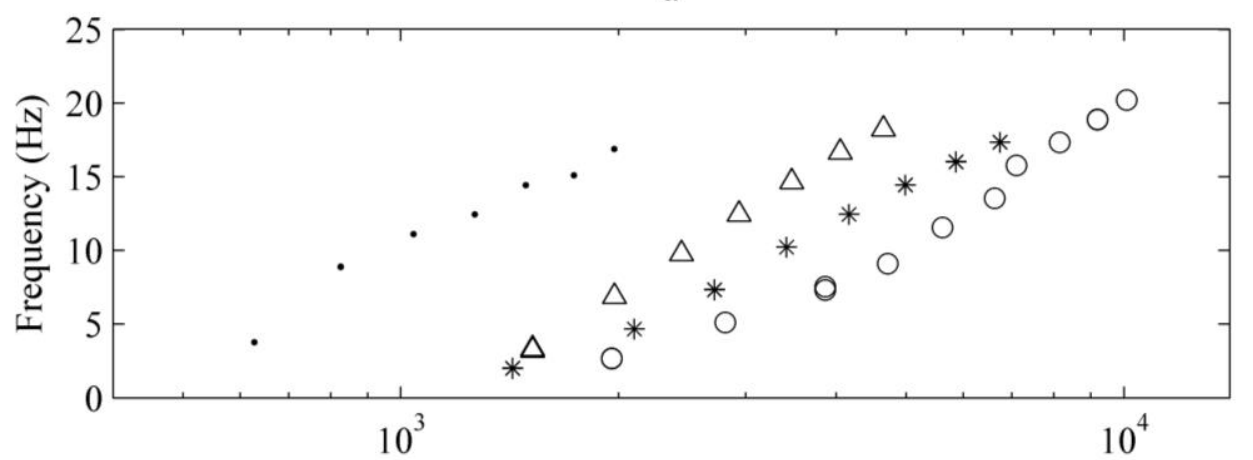

$\mathrm{b}$

$\operatorname{Re}$
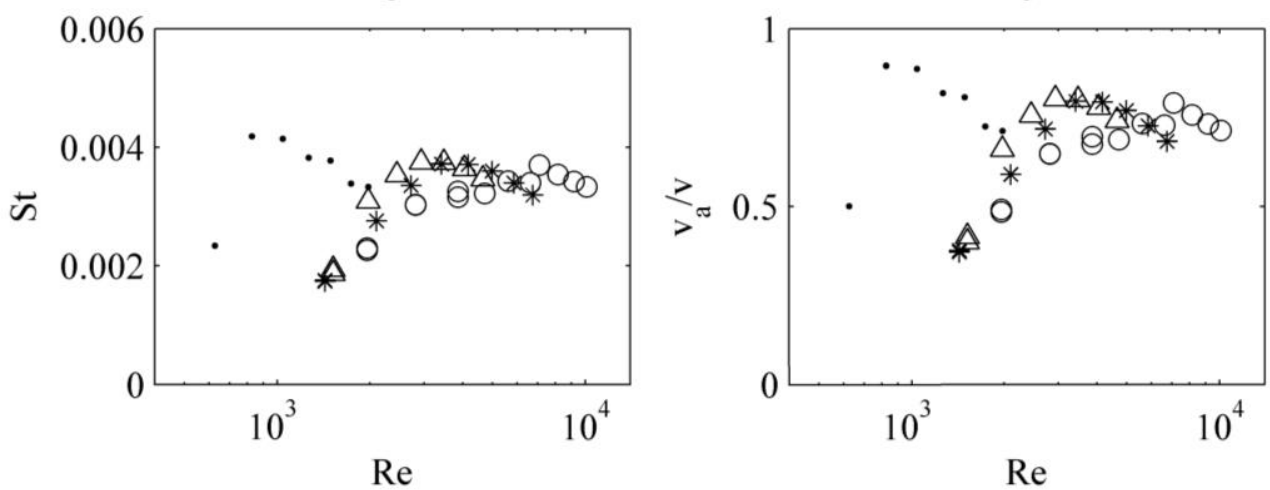

$\operatorname{Re}$

Figure 6 - Base design: effect of flow rate and viscosity $\left(01.00 \mathrm{~mm}^{2} / \mathrm{s}, * 1.35 \mathrm{~mm}^{2} / \mathrm{s}, \Delta 1.89\right.$ $\mathrm{mm}^{2} / \mathrm{s}, \cdot 4.37 \mathrm{~mm}^{2} / \mathrm{s}$ ) on the (a) frequency response, (b) Strouhal Number, (c) Modified Strouhal number (ratio of feedback channel velocity to power jet velocity)

\subsection{Feedback Loop Width}

The width of the feedback channel controls the pressure recovery in the system [19]. Smaller feedback channel widths and larger feedback channel lengths produce slower oscillations (for air) as a result of greater attenuation of the pressure waves in the feedback channel [20]. Additionally, the rise time of the pressure differential across the feedback channel to induce switching increases for smaller widths [21].

As shown in Figure 7a it was observed that, for liquid kinematic viscosities from $1.002-1.891 \mathrm{~mm}^{2} / \mathrm{s}$ (see Table 2), oscillator widths of 2-3.5 $\mathrm{mm}$ produced stable and welldefined oscillations with no definitive effect of width observable. For a liquid kinematic viscosity of $4.373 \mathrm{~mm}^{2} / \mathrm{s}$, only feedback widths of 3-3.5 $\mathrm{mm}$ produced stable oscillations. Here it is likely that the combination of higher viscosity and smaller width resulted in increased shear stress within the feedback channel, inhibiting the momentum exchange required to induce flow switching.

For all liquid mixtures studied, a $4 \mathrm{~mm}$ feedback loop width was unable to induce any oscillations. This could be because this width causes the outer edge of the feedback loop to exceed the point at which the jet attaches to the wall, preventing the formation of a lowpressure region that is required to drive the oscillations. The optimal feedback loop width for an oscillator of the dimensions shown in Figure 2 appears to be 3-3.5 $\mathrm{mm}$.

Analysing the dimensionless frequency results in Figure $7 \mathrm{~b}$ and $\mathrm{c}$, two operating regimes can be distinguished for each of the oscillator widths. The reduction of $v_{a} / v$ following the maximum as $R e$ increases again may be the result of increased pressure losses in the feedback channel created by secondary flows. 
a

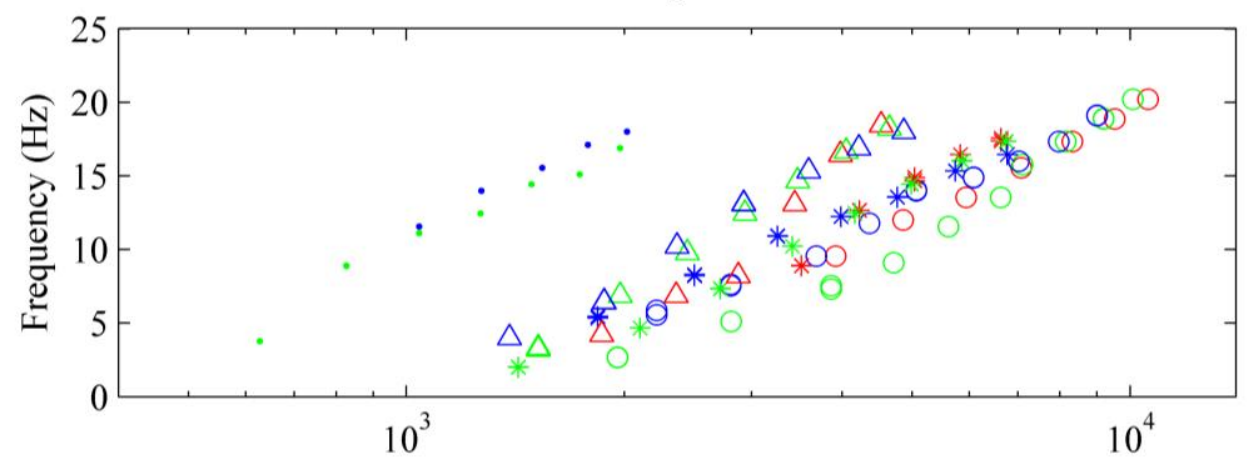

b

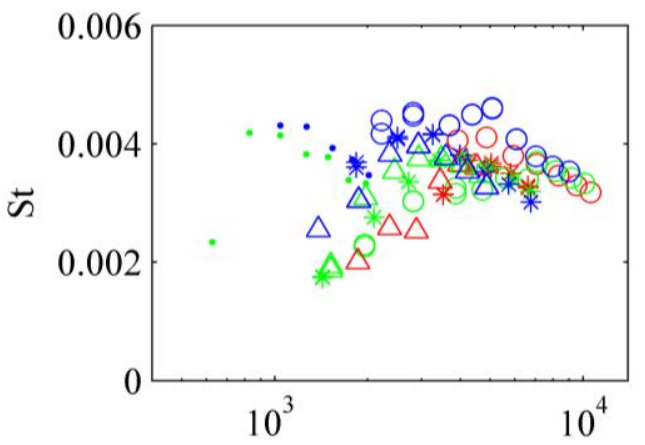

$\operatorname{Re}$

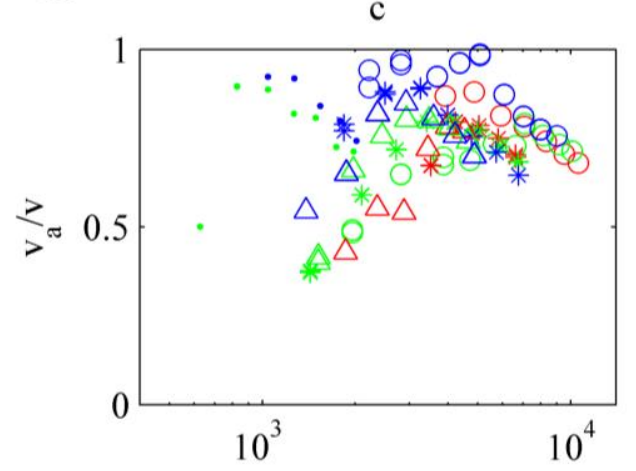

$\mathrm{Re}$

$\operatorname{Re}$

Figure 7 - Effect of feedback loop width $(2 \mathrm{~mm}, 3 \mathrm{~mm}, 3.5 \mathrm{~mm})$ at different liquid kinematic viscosities ( $01.00 \mathrm{~mm}^{2} / \mathrm{s}, * 1.35 \mathrm{~mm}^{2} / \mathrm{s}, \Delta 1.89 \mathrm{~mm}^{2} / \mathrm{s}, \cdot 4.37 \mathrm{~mm}^{2} / \mathrm{s}$ ) on the (a) frequency response, (b) Strouhal Number, (c) Modified Strouhal number (ratio of feedback channel velocity to power jet velocity)

\subsection{Feedback Loop Length}

The feedback loop length is a crucial design parameter according to the literature. For example, Tesař et al [11,22] have extensively reported the effects of feedback channel length for air and observed smaller switching frequencies at a fixed flow rate when increasing the feedback loop length. Arwatz et al $[16,20]$ also observed decreased switching frequencies at higher feedback lengths with air, and found that the onset of switching occurred earlier for smaller feedback channel lengths.

One of the limitations of this study was the size of the feedback loop length that could be tested. The lower limit $(101 \mathrm{~mm})$ was a result of keeping the inlet length $(32 \mathrm{~mm})$ constant, and the MiiCraft+ printer itself imposed an upper limit of $107 \mathrm{~mm}$. The Form1+ printer was used to create the $113 \mathrm{~mm}$ feedback length design.

Figure 8 shows the data collected when varying the feedback loop length. For water, the smallest feedback channel length $(101 \mathrm{~mm})$ produced higher switching frequencies than the other lengths $(107 \mathrm{~mm}$ and $113 \mathrm{~mm}$ ) for $R e<6000$. When $R e>6000$, the $107 \mathrm{~mm}$ length appeared to be optimal. Increasing the viscosity was found to reverse this trend, with the $40 \%$ glycerol solution (viscosity of $4.373 \mathrm{~mm}^{2} / \mathrm{s}$ ) showing the $101 \mathrm{~mm}$ length to produce higher frequencies at higher $R e$. The $113 \mathrm{~mm}$ feedback channel length produced results similar to the $107 \mathrm{~mm}$ length.

Figure $8 \mathrm{~b}$ presents the dimensionless frequency results $(S t)$ and again shows there are two distinct regimes. Here the maximum in $S t$ is more apparent than the results shown in Figure $7 \mathrm{~b}$. 


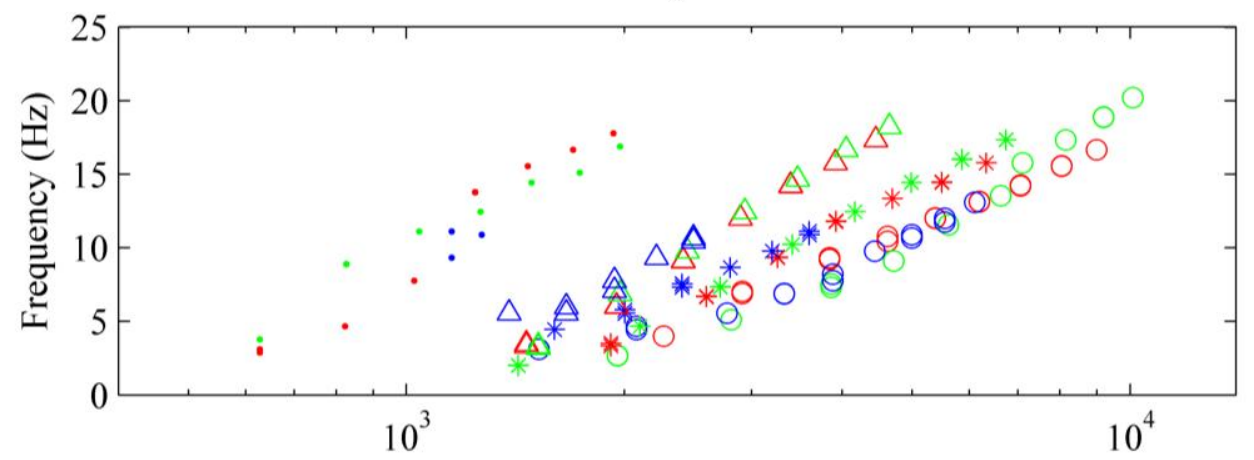

b

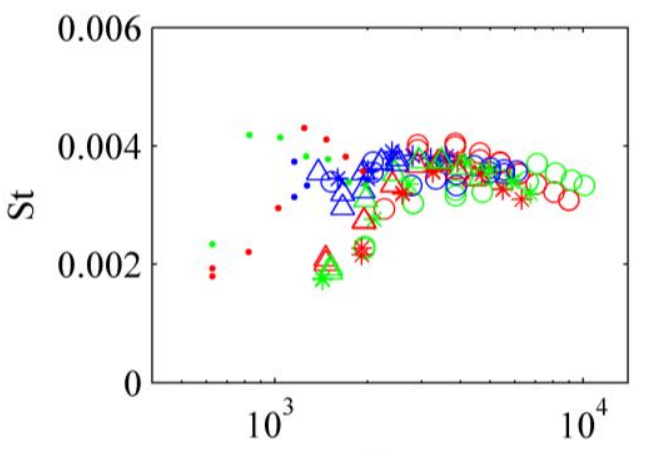

$\operatorname{Re}$

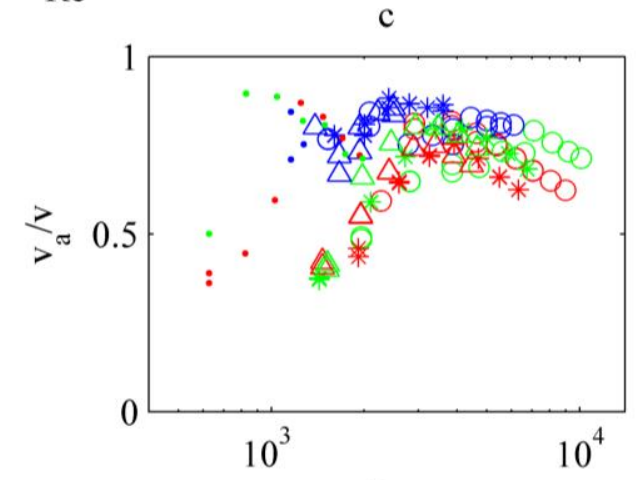

$\operatorname{Re}$

$\operatorname{Re}$

Figure 8 - Effect of feedback loop length $(101 \mathrm{~mm}, 107 \mathrm{~mm}, 113 \mathrm{~mm})$ at different liquid kinematic viscosities $\left(01.00 \mathrm{~mm}^{2} / \mathrm{s}, * 1.35 \mathrm{~mm}^{2} / \mathrm{s}, \Delta 1.89 \mathrm{~mm}^{2} / \mathrm{s}, \cdot 4.37 \mathrm{~mm}^{2} / \mathrm{s}\right)$ on the (a) frequency response, (b) Strouhal Number, (c) Modified Strouhal number (ratio of feedback channel velocity to power jet velocity)

\subsection{Splitter Distance}

Figure 9a shows the effect of splitter distance (distance from the power nozzle to the two outlet channels) on the switching frequencies. Here a more definitive effect was observed indicating that this is a more important design parameter. For liquid viscosities of 1.002 $4.373 \mathrm{~mm}^{2} / \mathrm{s}$, decreasing the splitter distance produced higher switching frequencies at the same $R e$. This has been previously observed in an air-operated oscillator [23].

There are three different types of processes that result in the switching phenomena observed in fluidic oscillators. These are the slow, rapid and load-controlled mechanisms [24]. Bistable amplifiers typically operate via the rapid and slow routes. Rapid switching occurs via direct momentum transfer to the power jet via the feedback channel. Slow switching requires the growth of a separation bubble via the transfer of pressure around a single feedback loop (Figure 1). Prior to switching, the power jet flows towards one outlet before deflecting to the other. By reducing the splitter distance this effect will be reduced causing the switching process to occur earlier in the bubble growth process.

Figure $9 \mathrm{~b}$ shows the dimensionless frequency $(S t)$ plotted against $R e$. Here, the strong effect of the splitter distance is apparent for each of the liquid viscosities. The $7 \mathrm{~mm}$ splitter distance exhibited the same maximum in $S t$ as discussed in the previous results (Figure $7 \mathrm{~b}$ and Figure 8b). In contrast, the $10 \mathrm{~mm}$ splitter distance showed a continual increase in $S t$ followed by a levelling off as $R e$ was increased, while the $5 \mathrm{~mm}$ distance showed only a decrease in $S t$ when increasing $R e$.

Analysing the modified Strouhal number (Figure 9c), it can be seen that at the $5 \mathrm{~mm}$ splitter distance the velocity within the feedback loop exceeds the velocity of the emerging jet from the power nozzle by a significant margin. The modified Strouhal number (equation 2) is applicable only when the switching process occurs on a much faster timescale than the growth of the separation bubble [2]. Therefore, even if this assumption is not valid here, the 
implication is the feedback velocity is still in excess of the power jet velocity. It has been shown that the concave wall within the oscillating chamber causes the formation of a second vortex that stabilises the flow switching process [10]. This secondary vortex may also aid the flow switching process in some capacity and could also be responsible for the frequency gain.
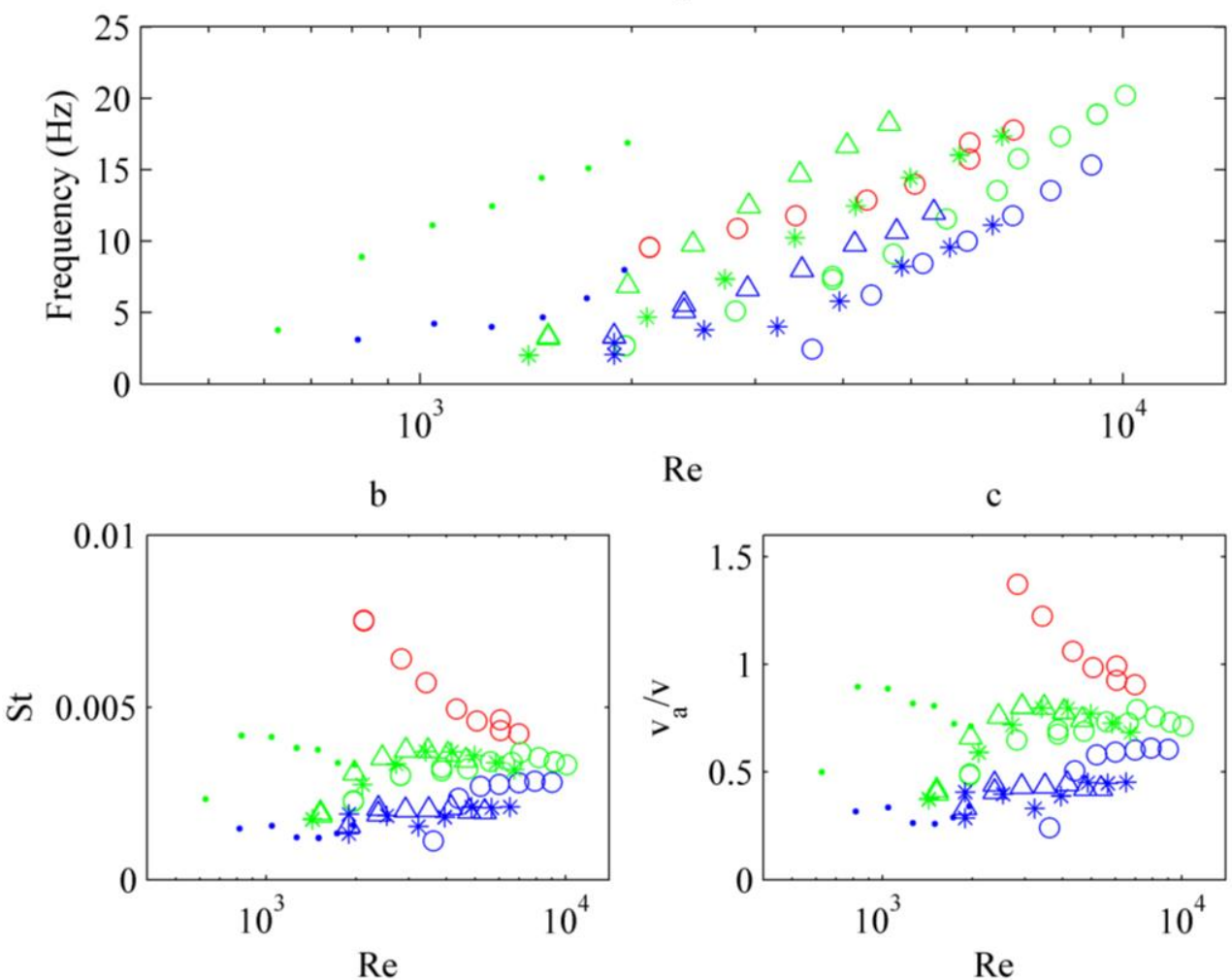

Figure 9 - Effect of splitter distance $(5 \mathrm{~mm}, 7 \mathrm{~mm}, 10 \mathrm{~mm})$ at different liquid kinematic viscosities ( $01.00 \mathrm{~mm}^{2} / \mathrm{s}, * 1.35 \mathrm{~mm}^{2} / \mathrm{s}, \Delta 1.89 \mathrm{~mm}^{2} / \mathrm{s}, \cdot 4.37 \mathrm{~mm}^{2} / \mathrm{s}$ ) on the (a) frequency response, (b) Strouhal Number, (c) Modified Strouhal number (ratio of feedback channel velocity to power jet velocity)

\subsection{Power Nozzle Converging Length}

Figure 10a shows the frequencies obtained when changing the convergence length of the power nozzle. The $5 \mathrm{~mm}$ converging length produced higher frequencies at lower flow rates for water, whilst the larger convergence length $(25 \mathrm{~mm})$ produced the highest frequencies for water for $R e>6000$. This suggests that maintaining laminar characteristics of the jet emerging from the power nozzle is important for flow switching. This is because a turbulent jet may disrupt the formation of the separation bubble.

Generally, the frequencies of the different converging lengths at high flow rate varied between 17 and $20 \mathrm{~Hz}$. Campo et al [25] investigated the wall angle in a double feedback bistable oscillator and found that it had little influence over the switching frequency. They found that changing the wall angle only shifted the standing vortex within the mixing chamber, but the growth mechanism remained unchanged. Changing the nozzle convergence length here may similarly alter the wall attachment point of the power jet, or may influence the size/shape of the secondary stabilisation vortex.

As with the previous results, Figure 10b/c shows two regimes of Strouhal number as $R e$ is increased. 
a

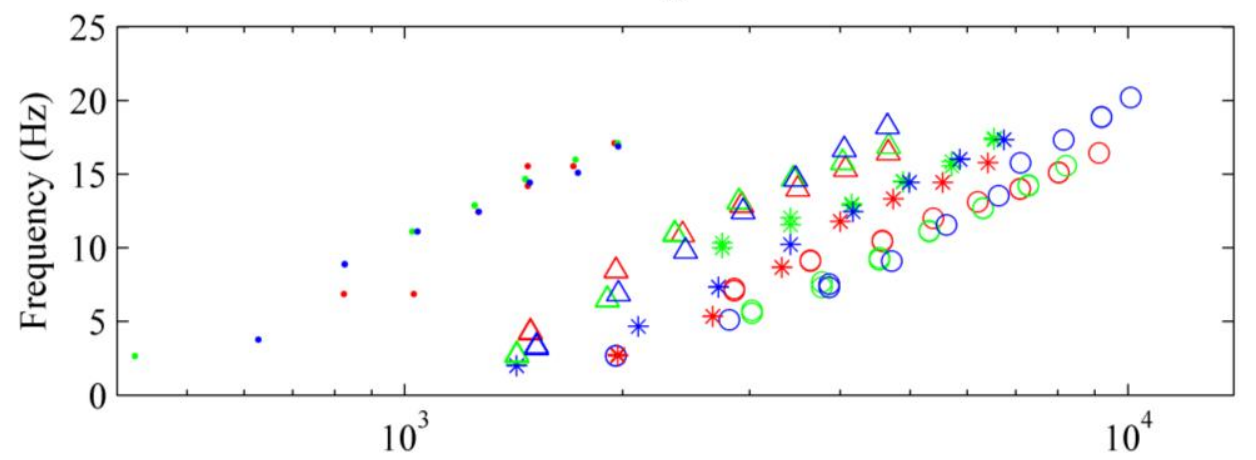

b

$\operatorname{Re}$
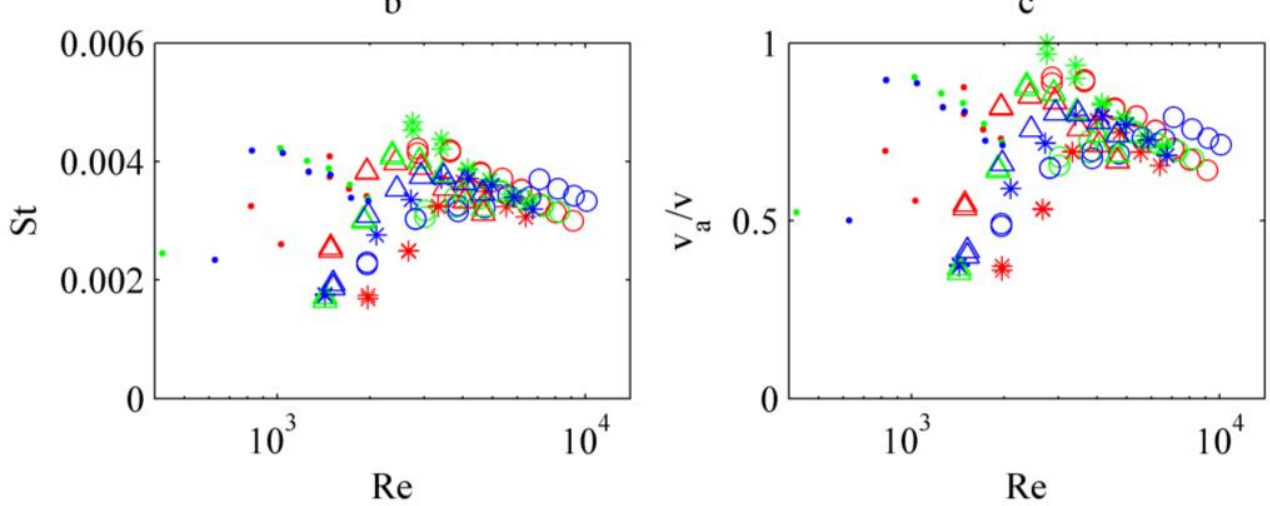

Figure 10 - Effect of Nozzle Convergence $(5 \mathrm{~mm}, 15 \mathrm{~mm}, 25 \mathrm{~mm})$ at different liquid kinematic viscosities ( $\left.01.00 \mathrm{~mm}^{2} / \mathrm{s}, * 1.35 \mathrm{~mm}^{2} / \mathrm{s}, \Delta 1.89 \mathrm{~mm}^{2} / \mathrm{s}, \cdot 4.37 \mathrm{~mm}^{2} / \mathrm{s}\right)$ on the (a) frequency response, (b) Strouhal Number, (c) Modified Strouhal number (ratio of feedback channel velocity to power jet velocity)

\subsection{Inlet Length}

Decreasing the total inlet nozzle length from $32 \mathrm{~mm}$ to $22 \mathrm{~mm}$ (with a nozzle convergence length of $15 \mathrm{~mm}$ ) was found to prevent any flow switching from occurring. It is possible that this reduced length does not allow sufficient development of a steady laminar profile prior to entering the power nozzle. Thus, this may further imply that laminar characteristics of the power jet are preferable. One method to improve the laminar characteristics for future study is the incorporation of straighteners prior to the power nozzle.

\subsection{Outlet Channel Angle}

The effect of changing the angle between the two outlet channels is shown in Figure 11. Angles of $18^{\circ}$ and $24^{\circ}$ produced higher oscillations than an angle of $12^{\circ}$ at the same flow rate for all fluid viscosities investigated. This disagrees with a CFD study (Campo et al, 2015) that found that the frequency decreased linearly with an increase in outlet angle. The angle influences the magnitude and position of the stagnation pressure, which influences the frequency. However, this CFD study was based on the double feedback loop design, rather than the single feedback design used here. In these designs, momentum transfer to the power jet is primarily responsible for causing the switching between outlets. In these double loop designs, increasing the angle here may reduce the feedback flow rate.

For the single feedback loop design, the larger outlet angle moves the wall attachment point downstream. Therefore, there may be an optimum angle where the pressure in the separation bubble formed from wall attachment is minimised, enabling faster momentum transfer around the feedback loop. After this critical angle a decrease in frequency would be expected, but this was not observed in the range of angles investigated here.

Based on the results in Figure $11 \mathrm{~b}$, it appears the optimal angle is between $18^{\circ}$ and $24^{\circ}$, as this generally produced higher values of St. However, the St appears to become invariant to the angle at higher flow rates. 


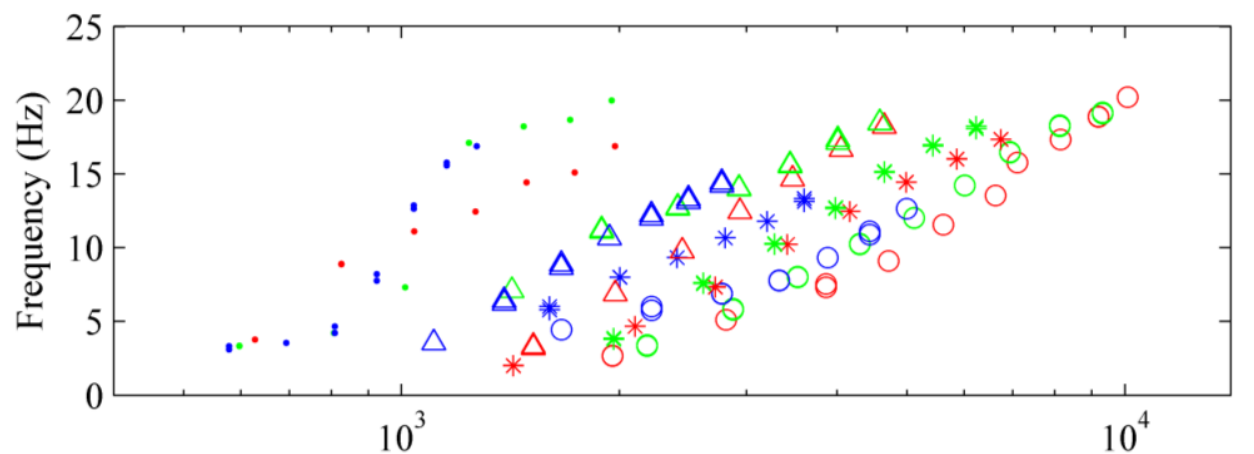

b

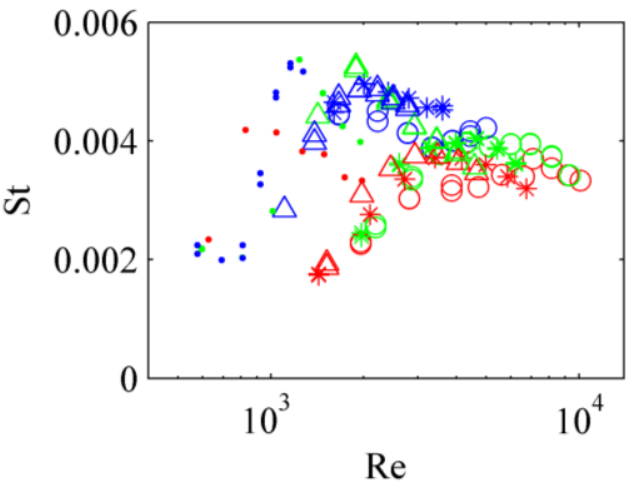

$\operatorname{Re}$

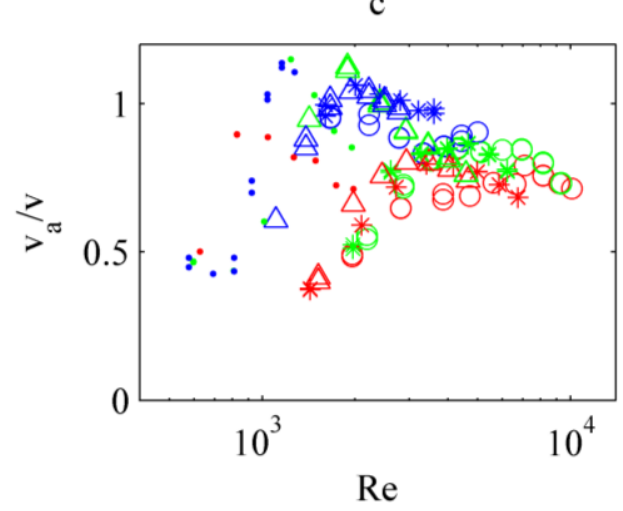

Figure 11 - Effect of Outlet Channel Angle $\left(12^{\circ}, 18^{\circ}, 2^{\circ}\right)$ at different liquid kinematic viscosities $\left(01.00 \mathrm{~mm}^{2} / \mathrm{s}, * 1.35 \mathrm{~mm}^{2} / \mathrm{s}, \Delta 1.89 \mathrm{~mm}^{2} / \mathrm{s}, \cdot 4.37 \mathrm{~mm}^{2} / \mathrm{s}\right)$ on the (a) frequency response, (b) Strouhal Number, (c) Modified Strouhal number (ratio of feedback channel velocity to power jet velocity)

\subsection{Feedback Loop Orientation}

One of the limits of the horizontal feedback loop configuration is the requirement to maintain a minimum inlet length. Therefore, several variations of vertical loop oscillators were also fabricated and validated to make the feedback loop length independent of the inlet length. The first vertical loop design used the same dimensions as the base design in Figure 2. A second version then used a shorter vertical feedback loop length $(75 \mathrm{~mm})$ whilst maintaining the other dimensions. However, both of these designs failed to provide the desired oscillatory response.

Three further variants (Figure 12) were then designed and printed to incorporate a $3 \times 3 \mathrm{~mm}$ feedback channel (width vs depth) to eliminate any design bias by using vertical loops. It was found that only the designs in Figure 12a and c provided oscillations, with the frequencies obtained shown in Figure 13. Based on these results, it was concluded that the vertical feedback loop might introduce an additional z-axis velocity component to the oscillating chamber that destabilises the secondary vortex. This behaviour can then be overcome using small straight sections prior to the feedback loop. 


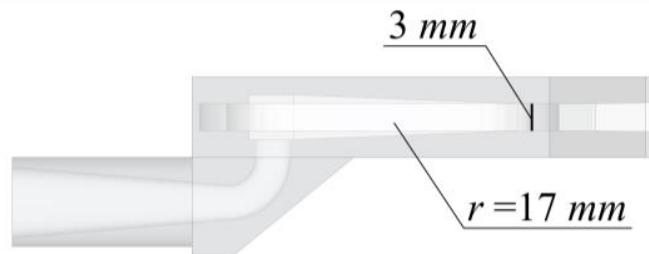

(b)

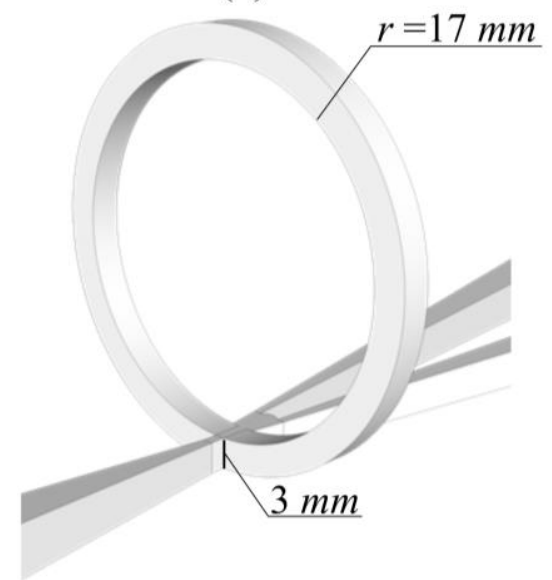

(a)

(c)

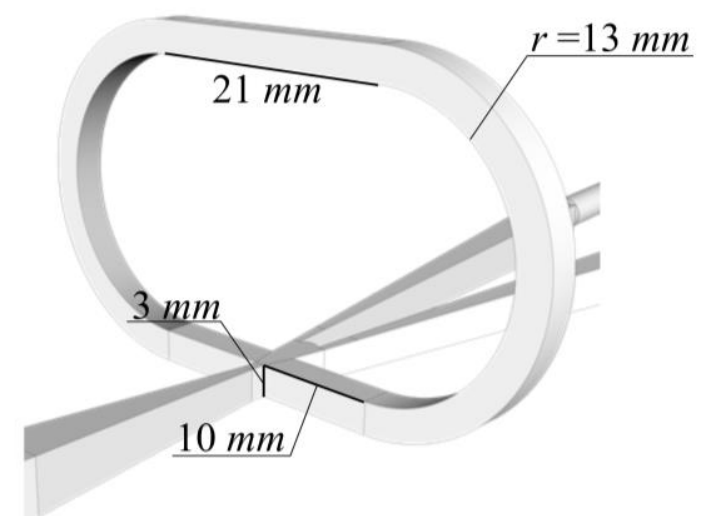

Figure 12 - (a) Horizontal loop configuration with narrowing of the oscillating chamber allowing for $3 \times 3 \mathrm{~mm}$ feedback channel, (b) vertical loop configuration with narrowing of the oscillating chamber allowing for $3 \times 3 \mathrm{~mm}$ feedback channel, and (c) vertical loop configuration with straight section and narrowing of the oscillating chamber allowing for $3 \times 3$ mm feedback channel

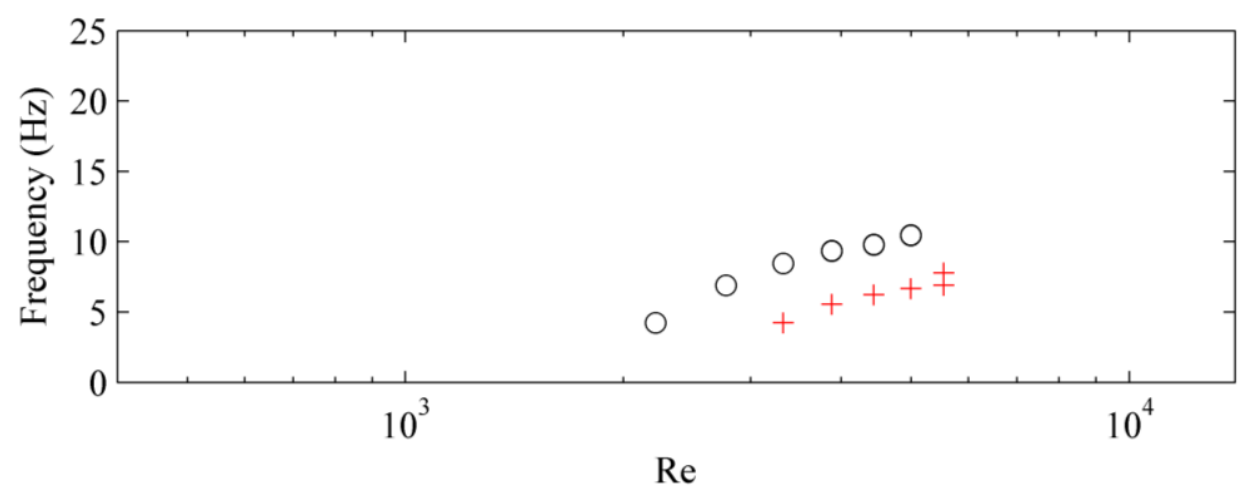

Figure 13 - Comparison of horizontal feedback loop configuration (०) and vertical feedback loop with straight section configuration $(+)$ using deionised water

\subsection{Collated Responses}

Figure 14 collates all recorded frequencies grouped via the selection of splitter distance. Here it can be seen that the choice of splitter distance dominates the frequencies for $R e<6000$. Higher frequencies are obtained using the shortest splitter distance $(5 \mathrm{~mm})$ whilst the onset $R e$ for flow switching is lower for the $7 \mathrm{~mm}$ splitter distance design. The lowest onset flow rate corresponds to using an outlet channel angle of $24^{\circ}$. The choice of geometric parameter appears to be less important at higher $R e$. 
a
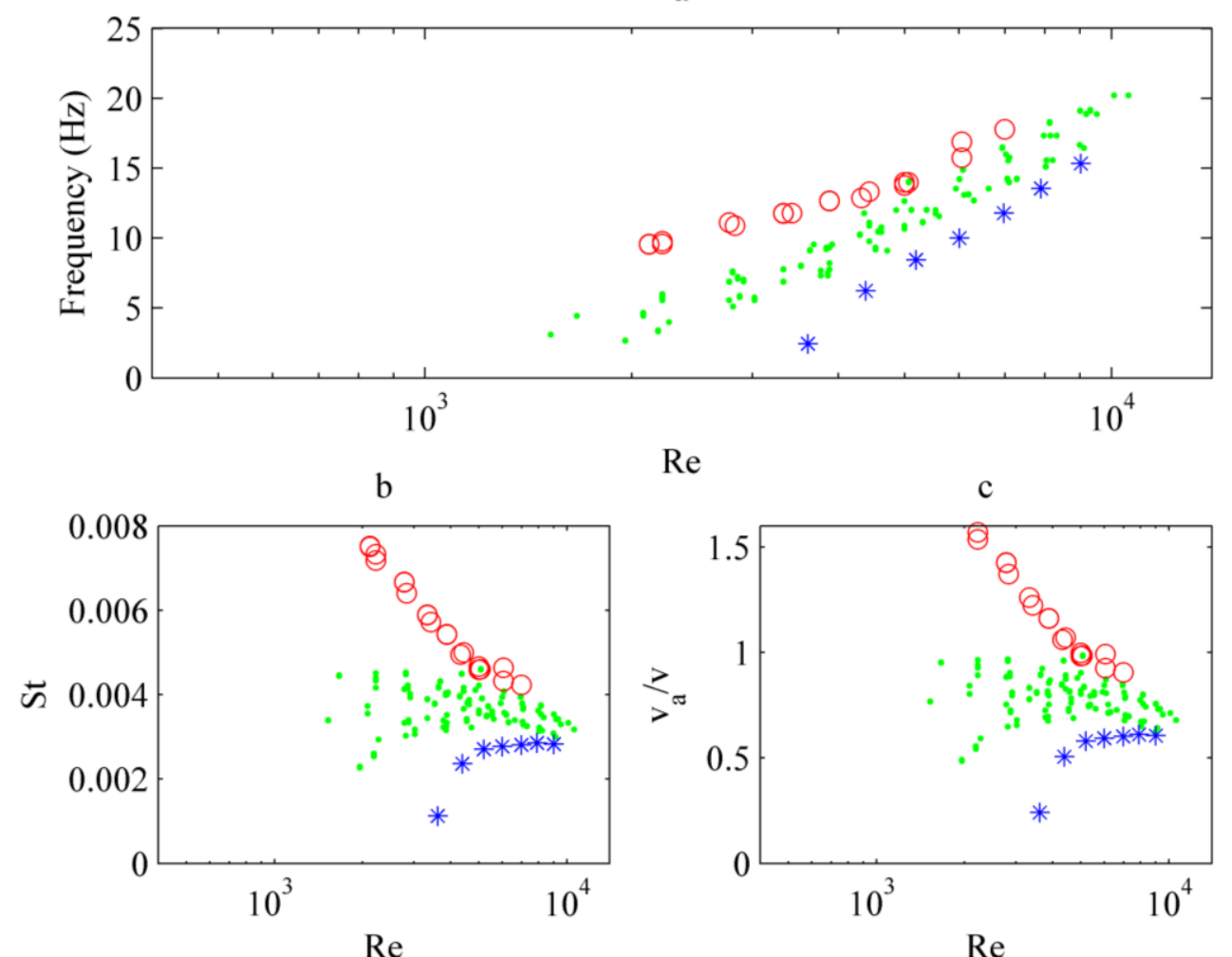

$\operatorname{Re}$

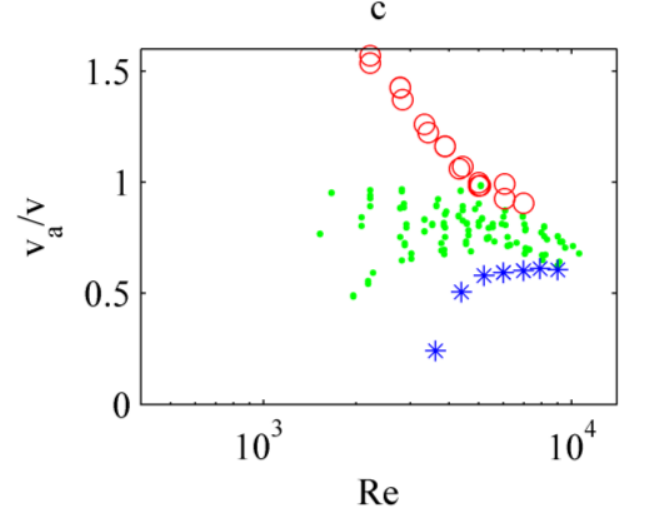

Figure 14 - Frequency response of all design variants for de-ionised water ( $\circ 5 \mathrm{~mm}$ splitter distance, $\cdot 7 \mathrm{~mm}$ splitter distance, $* 10 \mathrm{~mm}$ splitter distance) (a) frequency response, $(b)$ Strouhal Number, (c) Modified Strouhal number (ratio of feedback channel velocity to power jet velocity)

\section{Conclusions}

The design of liquid-based fluidic oscillators has been studied systematically using 3D printed prototypes for the first time. Within the parameter space explored in this study, switching frequencies of 2-22 $\mathrm{Hz}$ were obtained; with the highest flow-switching frequencies observed at high $R e$. It was found that the frequencies produced by all design variants converged at the higher flow rates $(R e>12,000)$, but for lower flow rates the seven geometric parameters did affect the frequencies obtained.

The splitter distance was found to have the greatest influence on the switching frequency, with higher frequencies produced at smaller splitter distances $(5 \mathrm{~mm})$. Additionally, larger outlet channel angles $\left(18-24^{\circ}\right)$ were found to produce slightly higher switching frequencies. The feedback loop width also influences the ability of these devices to oscillate. The smallest width $(2 \mathrm{~mm})$ was found to increase the shear stress, preventing oscillations from initiating for higher liquid viscosities, while the larger width $(4 \mathrm{~mm})$ completely inhibited oscillations. This may be because the larger width prevents the jet from attaching to one of the adjacent walls. Feedback channel widths of 3-3.5 $\mathrm{mm}$ were found to provide reliable oscillations regardless of the viscosity (up to $4.37 \mathrm{~mm}^{2} / \mathrm{s}$ ). Other geometric factors that inhibited flow switching were: shortening the inlet zone length from $32 \mathrm{~mm}$ to $22 \mathrm{~mm}$, and changing the orientation of the feedback loop from horizontal to vertical. Factors that did not greatly affect the frequency response were the convergence length of the power nozzle (5-25 $\mathrm{mm})$ and the feedback loop length (101-113 $\mathrm{mm}$ ).

Changing the viscosity of the liquid in the range of $1.00-4.37 \mathrm{~mm}^{2} / \mathrm{s}$ did not significantly affect the flow switching frequencies. However, liquids with viscosities of 29.8 and $75.9 \mathrm{~mm}^{2} / \mathrm{s}$ were unable to generate any oscillations in the parameter space investigated here. The frequencies were mainly influenced by the flow rates, with higher flow rates producing faster oscillations. 
Based on the literature and experimental results in this study, the following governing phenomena for flow switching can be described.

- Wall attachment. Controlled by either the Coandă effect or surface tension (or both), attachment of the power jet to one of the adjacent walls is necessary in order to cause the flow to exit predominately from one of the outlets at a time. The position of the wall attachment point of the jet may also influence the stagnation pressure, and consequently the driving force for flow switching. The controlling parameters are: feedback loop width, outlet channel angle and nozzle convergence length.

- Jet turbulence. Maintaining laminar characteristics of the emerging jet from the power nozzle may favour oscillations by improving adherence to the adjacent walls. The most consequential parameters here are the power nozzle convergence length and total inlet length.

- Separation bubble size. A low-pressure region (vortex) forms behind the wall attachment point. Growth of this low-pressure zone provided by the feedback flow controls the transition of the flow between the two outlets; the splitter distance predominately governs the bubble growth size needed for oscillations.

- Secondary vortex. The use of a concave wall opposite the power nozzle produces a second vortex that stabilises the power jet. The splitter distance and orientation of the feedback loop affect the formation of this vortex. In this study, there may be evidence that introducing an additional z-axis velocity component in the oscillating chamber destabilises this flow and prevents oscillations from occurring.

- Feedback flow regime. Flow switching frequency is mainly governed by the flow around the feedback channel. The development of secondary flow structures in this channel may account for limiting the frequencies at higher flow rates. Additionally, higher shear stresses in the feedback channel seem to inhibit oscillatory behaviour. Governing parameters here are the feedback channel width and length.

\section{Acknowledgements}

Financial support from EPSRC (EP/L504828/1) to author J.R. McDonough is gratefully acknowledged. Additional thanks to Simon Daley of CEAM is also warranted for electrical support and advice.

\section{Nomenclature}

$b \quad$ Power nozzle width $(m)$

$d \quad$ Feedback loop width $(m)$

De Dean number $(=\sqrt{d / r} R e)$

$f \quad$ Flow switching frequency $(\mathrm{Hz})$

$l \quad$ Feedback loop length $(m)$

$r \quad$ Feedback loop inner radius $(m)$

Re Reynolds number $(=v d / v)$

St $\quad$ Strouhal number $(=f b / v)$

$v \quad$ Liquid velocity emerging from the power nozzle $(\mathrm{m} / \mathrm{s})$

$v_{a} \quad$ Liquid velocity in the feedback loop $(\mathrm{m} / \mathrm{s})$

\section{Greek Letters}

$v \quad$ Kinematic viscosity $\left(\mathrm{m}^{2} / \mathrm{s}\right)$

\section{References}

[1] W.B. Zimmerman, B.N. Hewakandamby, V. Tesař, H.C.H. Bandulasena, and O.A. 
Omotowa, "On the design and simulation of an airlift loop bioreactor with microbubble generation by fluidic oscillation," Food and Bioproducts Processing, vol. 87, no. 3, pp. 215-227, 2009.

[2] V. Tesař, S. Zhong, and F. Rasheed, "New fluidic-oscillator concept for flow-separation control," AIAA Journal, vol. 51, no. 2, pp. 397-405, 2013.

[3] G. Raman and S. Raghu, "Cavity resonance suppression using miniature fluidic oscillators," AIAA Journal, vol. 42, no. 12, pp. 2608-2612, 2004.

[4] D. Guyot, B. Bobusch, C.O. Paschereit, and S. Raghu, "Active Combustion Control Using a Fluidic Oscillator for Asymmetric Fuel Flow Modulation," International Journal of Flow Control, vol. 1, no. 2, pp. 155-166, 2009.

[5] B.C. Bobusch, R. Woszidlo, J.M. Bergada, C.N. Nayeri, and C.O. Paschereit, "Experimental study of the internal flow structures inside a fluidic oscillator," Exp Fluids, vol. 54, p. 1559, 2013.

[6] C. Xu and Y. Chu, "An oscillating feedback microextractor with asymmetric feedback channels," Chemical Engineering Journal, vol. 253, pp. 438-447, 2014.

[7] C. Xu and Y. Chu, "Experiment al Study on Oscillating Feedback Micromixer for Miscible Liquids Using the Coanda Effect," AIChE Journal, vol. 61, no. 3, pp. 10541063, 2015.

[8] J.W. Gregory, J.P. Sullivan, G. Raman, and S. Raghu, "Characterization of the Microfluidic Oscillator," AIAA Journal, vol. 45, no. 3, pp. 568-576, 2007.

[9] V. Tesař, "High-frequency fluidic oscillator," Sensors and Actuators A, vol. 234, pp. 158-167, 2015.

[10] V. Tesař and H.C.H. Bandalusena, "Bistable diverter valve in microfluidics," Exp Fluids, vol. 50, pp. 1225-1233, 2011.

[11] V. Tesař, C.H. Hung, and W.B. Zimmerman, "No-moving-part hybrid-synthetic jet actuator," Sensors and Actuators A, vol. 125, pp. 159-169, 2006.

[12] P. Stonestreet and P.M.J. Van Der Veeken, "The effects of oscillatory flow and bulk flow components on residence time distribution in baffled tube reactors," Trans IChemE, vol. 77, no. Part A, pp. 671-684, 1999.

[13] A.N. Phan and A. Harvey, "Development and evaluation of novel designs of continuous mesoscale oscillatory baffled reactors," Chemical Engineering Journal, vol. 159, pp. 212-219, 2010.

[14] X. Ni, G. Brogan, A. Struthers, D.C. Bennett, and S.F. Wilson, "A systematic study of the effect of geometrical parameters on mixing time in oscillatory baffled columns," Trans IChemE, vol. 76, no. Part A, pp. 635-642, 1998.

[15] M. Mack, R. Niehuis, and A. Fiala, "Parametric study of fluidic oscillators for use in active boundary layer control," in Proceedings of ASME Turbo Expo 2011, Vancouver, British Columbia, Canada, 2011.

[16] G. Arwatz, I. Fono, and A. Seifert, "Suction and Oscillatory Blowing Actuator," in IUTAM SYMPOSIUM ON FLOW CONTROL AND MEMS.: Springer, 2008, vol. 46, pp. 33-44.

[17] N.S. Cheng, "Formula for the Viscosity of a Glycerol-Water Mixture," Ind. Eng. Chem. Res., vol. 47, pp. 3285-3288, 2008.

[18] P.M. Ligrani, "A study of Dean Vortex Development and Structure in a Curved Rectangular Channel with Asepct Ratio of 40 at Dean Numbers up to 430," US Army Research Laboratory, Contractor Report ARL-CR-144, 1994.

[19] T. Sarpkaya, "The performance characteristics of geometrically similar bistable amplifiers," Dudley Knox Library, 1972.

[20] G. Arwatz, I. Fono, and A. Seifert, "Suction and Oscillatory Blowing Actuator," 2008.

[21] C.J. Campagnuolo, "Review of some fluidic oscillators," Washington DC, 1969. 
[22] W.B. Zimmerman, V. Tesař, and H.C.H. Bandulasena, "Efficiency of an aerator driven by fluidic oscillation. Part 1: Laboratory bench scale studies," 2009.

[23] B.W. Anderson, The analysis and design of pneumatic systems.: Wiley, 1967.

[24] C.J. Williams, "An investigation of the flow pattern and splitter switching mechanism of bistable fluidic amplifiers," University of Windsor, PhD Thesis 1974.

[25] D.D. Campo, J.M. Bergada, and V.D. Campo, "Preliminary study on fluidic actuators. Design Modifications," in International Conference on Mechanics, Materials,

Mechanical Engineering and Chemical Engineering, Barcelona, Spain, 2015, pp. 53-61. 